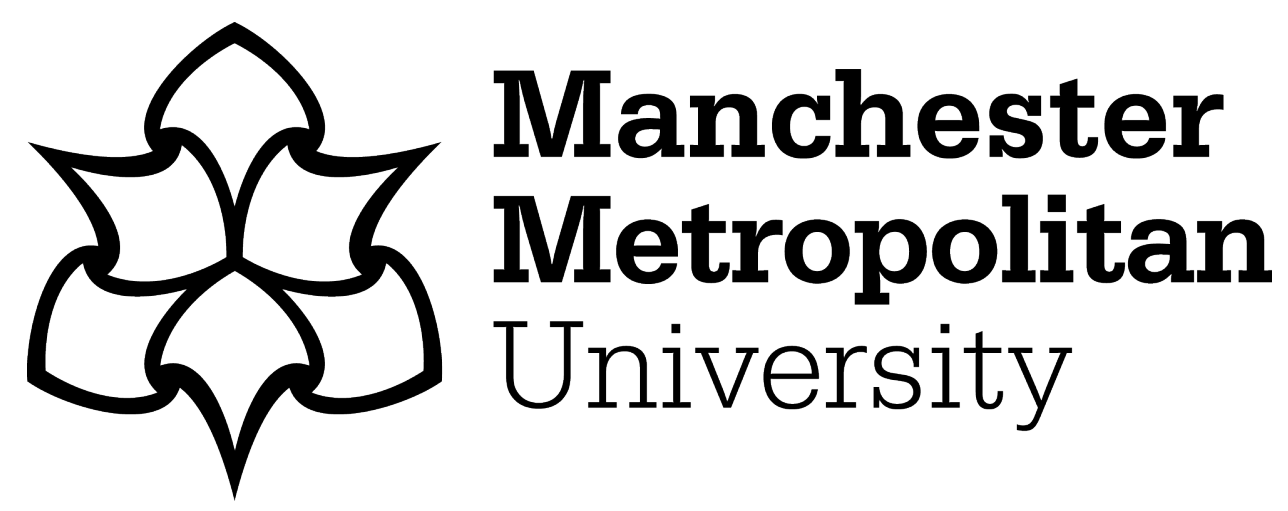

Alyamani, RAS and Murgatroyd, C (2018) Epigenetic Programming by EarlyLife Stress. Progress in Molecular Biology and Translational Science, 157. pp. 133-150. ISSN 1877-1173

Downloaded from: https://e-space.mmu.ac.uk/620971/

Version: Accepted Version

Publisher: Elsevier

DOI: https://doi.org/10.1016/bs.pmbts.2018.01.004

Usage rights: Creative Commons: Attribution-Noncommercial-No Derivative Works 4.0

Please cite the published version 


\title{
Epigenetic programming by early-life stress
}

\author{
Reema Abdulrahman S Alyamani and Chris Murgatroyd \\ School of Healthcare Science, Manchester Metropolitan University, Manchester, M1 \\ $5 G D U K$
}

\begin{abstract}
Studies show that adverse conditions during early life can increase vulnerability to mood disorders later in life. Around the early postnatal period the brain exhibits high plasticity enabling environmental signals to impact the trajectories of developing neuroendocrine circuits. Levels of environmental adversity in this early developmental period is able to shape the experience-dependent maturation of stress-regulating pathways leading to long-lasting alterations in stress responsivity during adulthood; a phenomenon often referred to as 'earlylife programming'. Current research is addressing the molecular mechanisms underlying this programming by which gene-environment interactions can predispose individuals toward psychopathology. Here we review key findings from animal and clinical studies examining the effects of the early environment in shaping development of the neuroendocrine regulation of stress and the role of epigenetic mechanisms in translating early-life conditions into long-lasting gene expression changes underpinning stress-related behaviors. Understanding how prenatal, postnatal and childhood experiences can give rise to lasting epigenetic marks conferring increased risk for mental disorders is becoming a major focus of molecular psychiatry and should pave new guidelines for therapeutic interventions.
\end{abstract}




\section{Introduction}

The close relationship between the quality of early life and mental health in later life is well described. Many human and rodent studies illustrate that aspects of the early environment can lead to dramatic changes in physical and mental development compromising altered cognition, mood and behaviour. In particular, stress and adverse conditions during early life periods of development can shape individual differences in vulnerability to stress-related disorders throughout life (for review see (Murgatroyd \& Spengler 2011c)). This raises the question of how environmental experiences become incorporated at the cellular and molecular level leading to long-term alterations in various neuronal functions, including stress responses and risk to mental disease.

To understand gene-environment interactions and to elucidate pathways through which early life experiences are able to program long-term responses, researchers in the neuroscience use animal models, in which the early environment can be manipulated in a controlled fashion, and clinical studies. Current work suggests that so-called epigenetic mechanisms of gene regulation, which alter the activity of genes without changing the order of their DNA sequence, could explain how early life experiences can leave lasting chemical marks on the brain and influence both physical and mental health later in life even when the initial trigger is long gone.

In this review we highlight important animal and human studies addressing epigenetic regulation of gene expression in sustaining the effects of early life experiences. We focus on studies that have investigated how adversity is able to shape biological stress systems, particularly the hypothalamic-pituitary-adrenal (HPA) system - an integral component of the body's stress response - leading to lasting alterations in stress responsivity (for review see (Murgatroyd \& Spengler 2011a). We discuss what we know about the function of epigenetic systems and their roles in programming brain development and disease in response to prenatal and postnatal environmental stressors.

\section{Early life adversity shapes later life stress responsivity}


Upon exposure to a stressor; the autonomic nervous system initiates a rapid and relatively short-lived " fight-or-flight" response, whilst the HPA axis is slower, instigating a more protracted response. Tight regulation of the HPA axis is core to the long-term control of systems governing stress responsivity. Following a stressor, the neuropeptides corticotropinreleasing hormone $(\mathrm{CRH})$ and arginine vasopressin $(A V P)$ are released from the paraventricular nucleus (PVN) of the hypothalamus that stimulate release of adrenocorticotrophic hormone $(\mathrm{ACTH})$ from the anterior pituitary which in turn acts on the adrenal cortex to release glucocorticoid hormones, i.e. cortisol (humans) corticosterone (animals). A negative feedback loop, through glucocorticoid receptors (GR) ensures efficient return to a homeostatic balance when it is no longer challenged (Figure 1). It has been shown that this negative feedback can become dysregulated, particularly following periods of chronic stress. This may critically impact the development of affective disorders; altered HPA activity is one of the most commonly observed neuroendocrine symptoms in major depressive disorder (for review see (Holsboer 2000)). Childhood stress is a strong predictor of impaired inhibitory feedback regulation of the HPA axis (for review see (Gonzalez 2013)) Studies in rodent models further support the concept that exposure to a chronic stressor can lead to long-term changes in HPA regulation and behavior stemming from changes in neuropeptide regulation (for review see (Murgatroyd \& Bradburn 2016)).

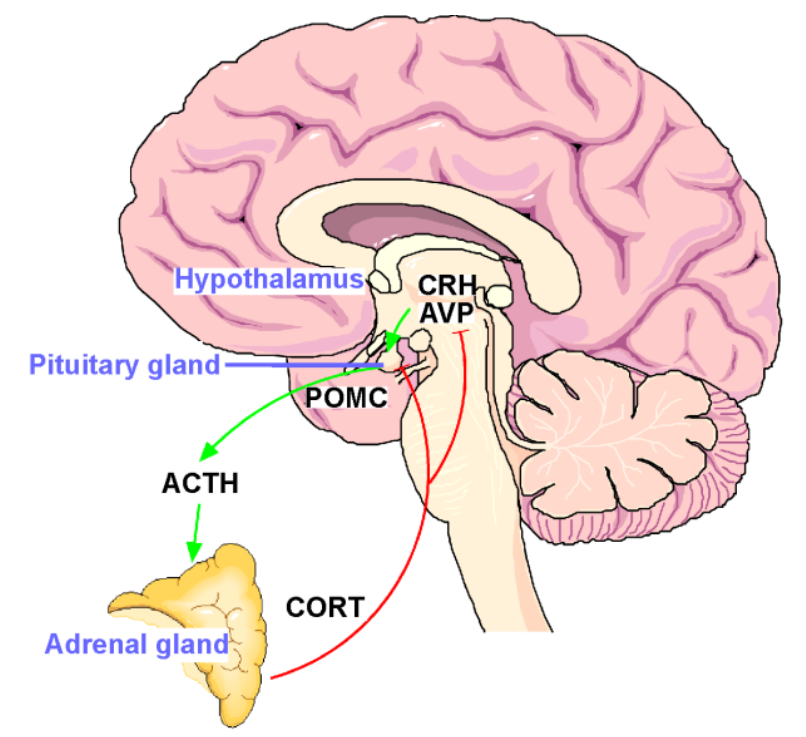


Figure 1. The HPA stress axis. The neuropeptides corticotrophin-releasing hormone (CRH) and arginine vasopressin (AVP) are expressed in the parvocelluar neurons of the hypothalamic nucleus paraventricularis. The joint release of $\mathrm{CRH}$ and AVP into the portal blood vessels leads to potent stimulation of anterior pituitary ACTH secretion and in turn of corticosterone from the adrenal glands. The activational effects of the HPA axis are counteracted by the inhibitory effects of glucocorticoid receptors expressed in the hippocampus, hypothalamus and anterior pituitary.

\section{Epigenetic mechanisms}

"Epigenetics" is a term used to describe the study of stable alterations in gene expression potential that arise during development and differentiation, and is under the influence of the environment (for review see (Doherty \& Roth 2016)). Genomes can therefore be considered to contain two layers of information: Firstly, there is the DNA sequence that is conserved throughout life and mostly identical in all cells of a body. Secondly, there are the epigenetic marks that are cell-specific and can be dynamic.

Epigenetic mechanisms enable regulation of a gene, independent of DNA sequence. DNA methylation and modification of core histones that package the DNA into chromatin represent the best-understood epigenetic marks. These marks are able to govern accessibility of the DNA to the machinery driving gene expression; inaccessible genes become silenced whereas accessible genes are actively transcribed.

These epigenetic processes enable a cell and organism to integrate intrinsic and environmental signals into the genome resulting in regulatory control of gene expression and thus facilitating adaptation. In this way, epigenetic mechanisms could be thought of as conferring plasticity to the hard-coded genome. In the context of the early life environment, epigenetic changes offer a plausible mechanism by which early experiences could be 
integrated into the genome as a kind of memory to program adult hormonal and behavioral responses.

\subsection{DNA Methylation}

DNA methylation, describes the addition of a methyl group to DNA at the cytosine side-chain in cytosine- guanine $(\mathrm{CpG})$ dinucleotides, known as a $\mathrm{CpG}$. The conventional view is that DNA methylation is a silencing mark and that, in general, the endpoint of DNA methylation is either long-term silencing or fine-tuning of gene expression potential. Hence, promoters and enhancer elements of transcriptionally active genes are usually methylated but may become silenced once targeted by DNA methylation. The insertion of methyl groups changes the appearance and structure of DNA, which may either: 1, directly block DNA binding of transcription factors or 2, attract factors that preferentially bind to methylated or unmethylated DNA to interfere with transcription factor accessibility (Figure 2A).

The vast majority (70-80\%) of all CpGs are methylated. Around $85 \%$ of these are located in repetitive sequences such as transposons that constitute around half of the human genome while the other $15 \%$ typically cluster within GC-rich regions known as "CpG islands". CpG islands are defined as regions of greater than $500 \mathrm{bp}$ that have cytosine/guanine content of greater than $55 \%$. Up to $60 \%$ of $\mathrm{CpG}$ islands are in the 5 ' regulatory (promoter) regions of genes. Although $\mathrm{CpG}$ islands, promoters and enhancer regions appear to attract most attention in regard to DNA methylation and gene transcription, it is becoming increasingly clear that methylation at other gene elements play important regulatory roles. Indeed genome-wide sequencing of DNA methylation patterns among different tissues are revealing the presence and locations of tissue-specific differentially methylated regions. Strikingly, many of these do not seem to occur in $\mathrm{CpG}$ islands themselves but tend to locate in regions next to $\mathrm{CpG}$ islands around $2 \mathrm{~kb}$ away within sequences of intermediate $\mathrm{CpG}$ density. These are now referred to as "CpG shores" (Gomez et al. 2016). Further regions can be divided up as "CpG shelves" as $2 \mathrm{~kb}$ regions extending from the shores while CpGs located yet further 
away from CpG islands are defined as being in "open sea" (Figure 2B). In contrast to repression-associated DNA methylation in promoters and enhancers, higher CpG methylation within gene bodies is being frequently observed in transcriptionally active genes perhaps suggesting a further functional role of DNA methylation in transcriptional regulation.

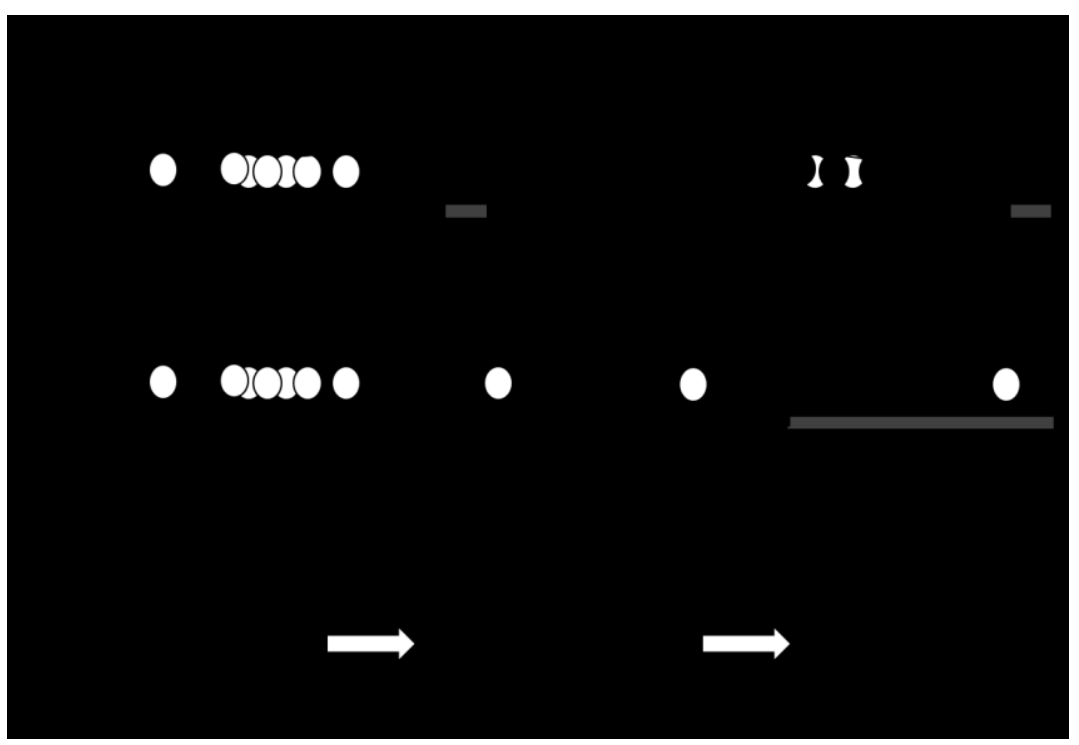

Figure 2. DNA methylation. A) DNA methylation can lead to gene repression through inhibiting transcription factor (TF) binding and recruitment of methylated DNA binding proteins (MBDs) and chromatin remodeling factors. B) GC-rich regions are known as $\mathrm{CpG}$ islands and areas around $2 \mathrm{~kb}$ away, within sequences of intermediate $\mathrm{CpG}$ density, are referred to as $\mathrm{CpG}$ shores. C) Dnmt1 is considered the primary maintenance methyltransferase while Dnmt3a and Dnmt3b are considered de novo methyltransferases important in establishing methylation.

The process of DNA methylation depends on a family of enzymes known as DNA methyltransferases (DNMTs). DNMT1 recognizes hemimethylated DNA and methylates appropriate cytosines in newly synthesized daughter strands formed during replication, acting as the maintenance DNMT. DNMT3A and DNMT3B can methylate unmethylated DNA supportive of their roles as de novo methylases (Figure $\mathbf{2 C}$ ). DNA methylation can also be a reversed. For example, during development there are global and gene-specific increases and decreases in levels of $5 \mathrm{mC}$. The TET family of enzymes can modify $5 \mathrm{mC}$ to 5 hydroxymethylcytosine $(5 \mathrm{hmC})$ as part of the processes of active demethylation. This 
modification, appears to be particularly abundant in brain and might allow dynamic regulation of DNA methylation.

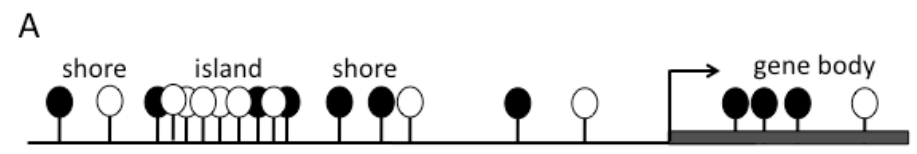

B

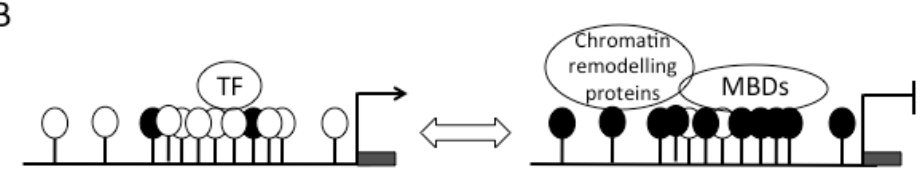

C

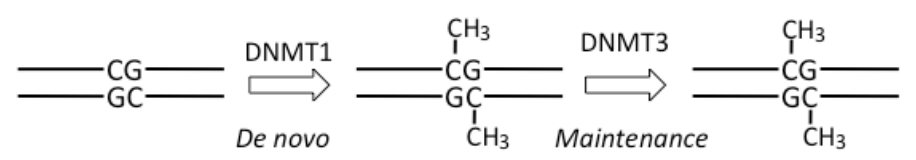

Figure 2 DNA methylation. (A) GC-rich regions are known as $\mathrm{CpG}$ islands and areas around $2 \mathrm{~kb}$ away, within sequences of intermediate CpG density, are referred to as $\mathrm{CpG}$ shores. (Black circle, methylated. White circle, unmethylated.) (B) DNA methylation (black circle) can lead to gene repression through inhibiting transcription factor (TF) binding and recruitment of methylated DNA-binding proteins (MBDs) and chromatin remodeling factors. (C) DNMT1 is considered the primary maintenance methyltransferase while DNMT3A and DNMT3B are considered de novo methyltransferases important in establishing methylation.

\subsection{Chromatin}

Chromatin is a complex of DNA wrapped around histone proteins. Histones can be modified at their $\mathrm{N}$-terminal tails by methylation, phosphorylation, acet- ylation, and ubiquitination as part of a histone signature serving to define accessibility to the DNA, i.e. densely packaged 'closed' chromatin (heterochromatin) in contrast to accessible 'open' chromatin (euchromatin). Histone acetylation is known to be a predominant signal for active chromatin configurations while some specific histone methylation reactions are associated with either gene silencing or activation. These are regulated through an array of histone modifying enzymes that catalyze the addition or removal of an array of covalent modifications. This 
'histone code' can be 'read' by specific proteins, which have particular domains that recognize certain histone modifications to produce an effect on gene expression. Chromatin remodeling plays a central role in gene regulation by providing the transcription machinery with dynamic access to an otherwise tightly packaged genome.

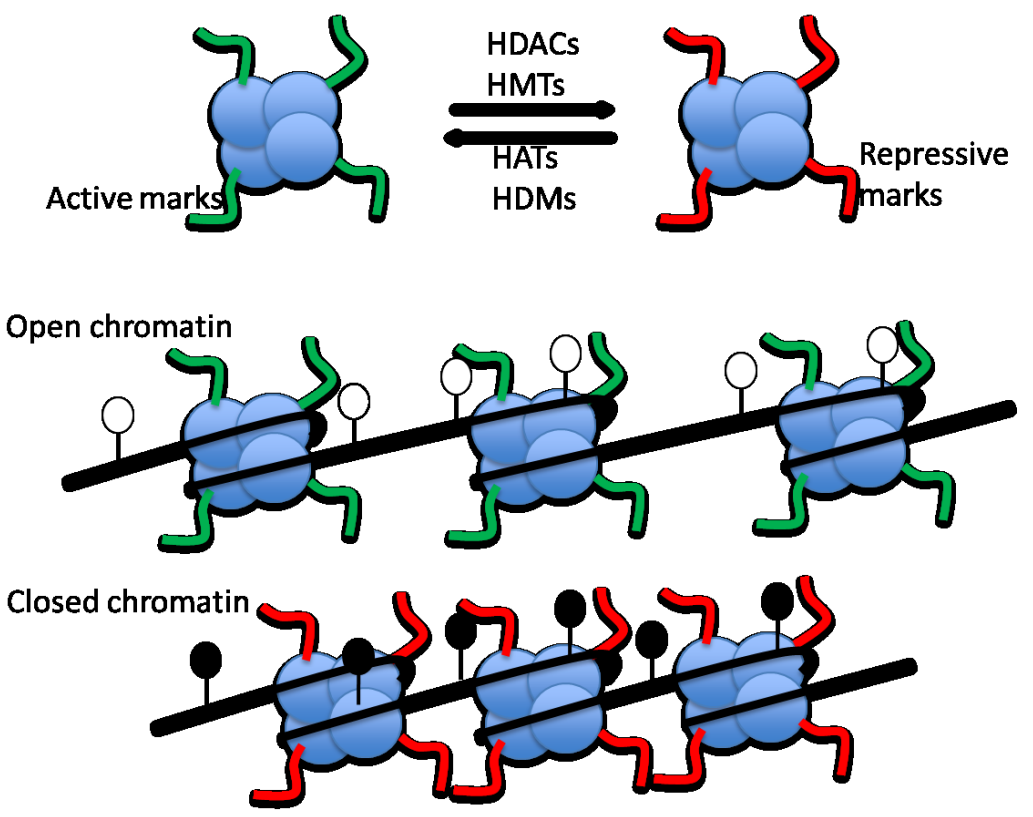

Figure 3. DNA wraps around histones to form a complex referred to as chromatin. The N-terminal tails of histones serve as sites for epigenetic marking by acetylation and methylation via chromatin remodelling enzymes (e.g. HDACs, HATs, HMTs and HDMs). DNA methylation refers to the chemical transfer of methyl groups to CpG acceptor sites through a class of enzymes known DNA methyltransferases (DNMTs). Active marks (e.g. histone acetylation and DNA hypomethylation) characterize "open" chromatin, while repressive marks (e.g. histone methylation and DNA hypermethylation) occur at "closed" chromatin.

\subsection{Non-coding RNA}

The vast majority of the human genome is transcribed, but only around $2 \%$ of these transcripts are translated into protein. However, many of these are nonetheless functional: 
known as non-coding RNA (ncRNA) they can function to regulate gene expression at the transcriptional and post-transcriptional level. These can be divided into two main groups; the short ncRNAs (<30 nts) and the long ncRNAs ( $>200 \mathrm{nts})$. The three major classes of short non-coding RNAs are microRNAs (miRNAs), short interfering RNAs (siRNAs), and piwiinteracting RNAs (piRNAs). Both major groups are shown to play a role in heterochromatin formation, histone modification, DNA methylation targeting, and gene silencing.

\section{Epigenetic programming by prenatal stress}

Aside from controlling constitutive gene expression, epigenetic mechanisms can also serve to fine-tune gene expression potential in response to environmental cues. Hence, it has been proposed that conditions of prenatal and postnatal environment can evoke changes in DNA methylation facilitating epigenetic programming of critical genes involved in regulating stress responsivity that may in turn manifest with neuroendocrine and behavioral symptoms in adulthood (Figure 1).

\subsection{Prenatal Stress}

In animal models prenatal stress has been shown to lead to various anxiety and depressivelike phenotypes (for review see (Babenko et al. 2015). It has been demonstrated that the long-term effects of early-life stress are mediated through alterations in the maternal and fetal HPA axes leading to exposure of the fetus to excess maternal glucocorticoids (Reynolds et al., 2013a) that may pass the placental barrier and disrupt fetal brain development.

An important factor in regulating supply of maternal glucocorticoids to the developing fetus is the placental enzyme, 11b hydroxysteroid dehydrogenase 2 (11b HSD-2), that deactivates cortisol (Stewart et al. 1995). It is important to control this placental diffusion as maternal glucocorticoid levels are up to 10-fold higher than in the fetus (Seckl 2001). Regulation of 
placental $11 \mathrm{~b}$ HSD-2 is altered in response to a number of maternal components including maternal undernutrition and protein deficiency (Bertram et al. 2001). Rodent studies have further shown it is up-regulated following acute stress in pregnancy (Welberg 2005) and that chronic stress and prenatal maternal anxiety causes a reduction of HSD11B2 placental expression that associated with increased $\mathrm{CpG}$ methylation of the HSD11B2 gene in the placenta (Jensen Peña et al. 2012).

The human HSD11B2 gene contains four CpG islands and studies have shown that its activity is regulated by methylation (for review see (Togher et al. 2014). In agreement with findings from the aforementioned animal models, study by O'Donnell et al. (O'Donnell et al. 2012)) revealed that prenatal Trait anxiety negatively correlated with placental $11 \beta-H S D 2$ mRNA expression. When testing infants, a study by Conradt et al. (Conradt et al. 2013) revealed that, intrauterine exposure to maternal depression negatively affected infant behaviour and this associated with increased methylation of the placental $11 \mathrm{~b}-H S D-2$ gene and the GR gene, NR3C1 while Appleton et al. (Appleton et al. 2015) also found that infants whose mothers experienced the greatest levels of socioe- conomic adversity during pregnancy had the lowest extent of methylation of the placental $11 b-H S D 2$ gene. This suggests that epigenetic regulation of $11 b-H S D 2$ links environmental cues transmitted from the mother to the foetus during pregnancy that could program the response to adverse postnatal environment via less exposure to glucocrticoids during development. Further studies have also identified differential epigenetic regulation of glucorticoids. For example, a study by Oberlander et al. (Oberlander et al., 2008) revealed that prenatal exposure to maternal depressed/anxious mood during the 3rd trimester of gestational development resulted in increased methylation of a $\mathrm{CpG}$-rich region in the promoter and exon1F of the NR3C1 gene in the cord blood of newborns, which correlated with salivary cortisol levels in infants at 3 months of age.

\subsection{Epigenetic programming by postnatal stress}


The early postnatal life is another sensitive period for stress programming effects. Numerous animal studies of impaired mother-infant interactions (such as maternal stress, separation or deprivation) during the early postnatal period have been shown to disrupt behaviours such as memory, learning, and anxiety- and depressive-like behaviors together with neuroendocrine dysregulation of the HPA axis (Murgatroyd \& Bradburn 2016).

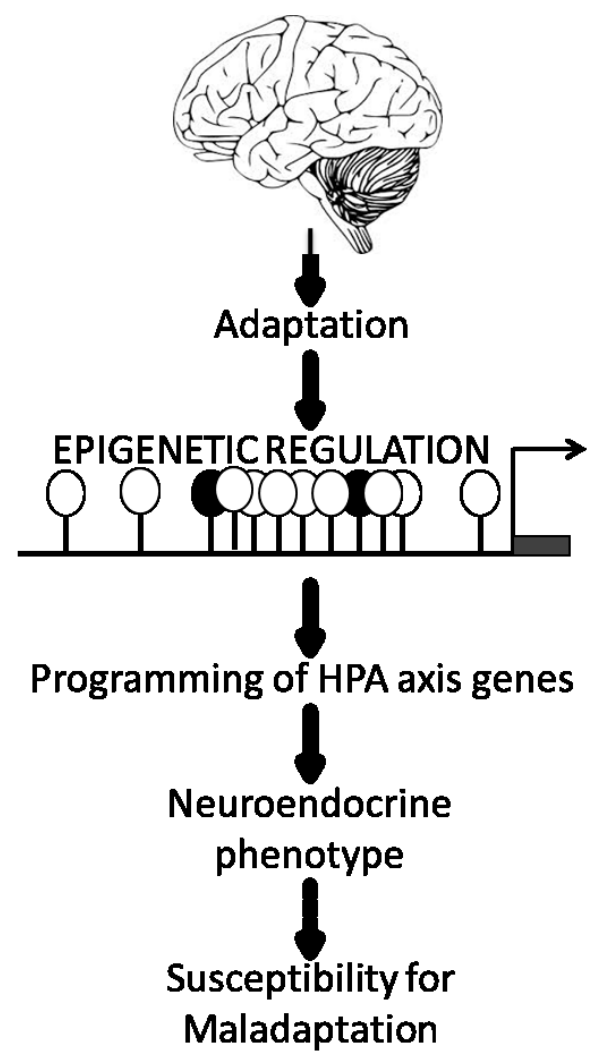

Figure 3. Early life experience can persistently alter expression levels of key genes through epigenetic marking which can underpin changes in behaviour, neuroendocrine and stress responsivity throughout later life. Collectively, this process is referred to as epigenetic programming. The nature of the environment throughout later life, in addition to the impact of biological processes associated with aging and genetic sex, may exacerbate the effects of programming established during early life resulting in increased vulnerability to mood disorders. 
A study of early-life adversity through the use of maternal separation in mice led to a sustained increase in expression of hypothalamic Avp in the paraventricular nucleus (PVN) that underlined elevated corticosterone secretion, heightened endocrine responsiveness to subsequent stressors, and altered HPA axis feedback inhibition. Importantly, this altered expression associated with reduced levels of DNA methylation in the PVN at particular CpG dinucleotides within an enhancer region important for Avp gene activity (Murgatroyd et al. 2009). Mechanistic investigations revealed that hypomethylation at this region reduced the ability of MeCP2 to bind and recruit further epigenetic machinery such as HDACs and DNMTs, supporting previous evidence for a role of MeCP2 as an epigenetic platform upon which histone and DNA modifications are carried out to confer transcriptional repression. Importantly, this hypomethylation was induced by neuronal depolarization triggering Ca2+dependent phosphorylation of MeCP2 causing MeCP2 to dissociate from the Avp enhancer resulting in a loss of both its repressing activity and its organization of DNA methylation and histone marks (Murgatroyd \& Spengler 2014).

Human studies reveal compelling evidence that adversity during early childhood may cause a range of physical and mental problems in adulthood including depressive-related disorders and alterations in HPA axis activity (for review see (Murgatroyd \& Spengler 2011b)). Importantly, a number of key studies demonstrate that the epigenetic programming seen in the early-life stress animal models may extrapolate to human studies. For example, McGowan and colleagues (McGowan et al. 2008) found hypermethylation of NR3C1 promoter among suicide victims with a history of abuse in childhood, but not among controls or suicide victims who did not suffer such early life stress. These data appear consistent with the previously described study at the same homologous region of the rat $N r 3 c 1$ promoter (Weaver et al., 2004). Numerous further studies support NR3C1 to be susceptible to alterations in DNA methylation, in response to early environmental stress (for review see (Smart et al. 2015). 
In a genome-wide study of promoter methylation in individuals with history of severe abuse during childhood, Labonte et al. (2012a) identified 362 differentially methylated promoters in hippocampal neurons from postmortem brain tissues of individuals with a history of severe childhood abuse compared to control subjects. Among these genes those involved in neuronal plasticity were most differentially methylated. More recently, Suderman et al. (2014) demonstrated that 997 gene promoters in whole blood DNA of adult subjects were differentially methylated in association with childhood abuse. Most of these genes are known to be involved in key cell signaling pathways linked to development and regulation of transcription. Provenc al et al. (2014), by analyzing the genome-wide promoter DNA methylation profiles in T cells from adult men, revealed that 448 distinct gene promoters were differentially methylated in persons with a history of parental physical aggression from 6 to 15 years of age compared to a control group. Functionally, most of these genes were previously demonstrated to play a substantial role in aggression and were enriched in biological pathways that are affected by behavior.

Numerous studies have demonstrated that childhood trauma increases risk factor for developing depression in adulthood, particularly in response to additional stress (for review see (Heim \& Binder 2012) ). The hypothesis is that neuroendocrine, particularly HPA activity, resultant from early-life stress could increase risk to develop depression in response to stress later in life. A study by Klengel et al. (Klengel et al. 2012) showed that an increased risk of developing adult stress-related psychiatric disorders associated with allele-specific, childhood trauma-dependent DNA demethylation at glucocorticoid response DNA elements of the FK506 binding protein 51 (FKBP5) gene, which encodes a co-chaperone which regulates GR sensitivity. The demethylation of $F K B P 5$ related to an increased stressdependent gene transcription followed by a long-term dysregulation of the stress hormone system and a global effect on immune functions and brain areas linked to stress regulation. 
Together this suggests that epigenetic processes in the brain could mediate the effects of the early environment on gene expression, and that stable epigenetic marks such as DNA methylation might then persist into adulthood to influence HPA axis activity.

\subsection{Interactions of prenatal and postnatal stress}

The foetal origins hypothesis of human disease that proposes that in utero environmental exposures lead to modifications in foetal development, which are adaptive where the subsequent postnatal environment is similar. Discontinuities between prenatal and postnatal environments create vulnerability. This effect is best exemplified in the associations of low foetal growth with diabetes and hypertension over several decades, that are thought to arise from foetal adaptations that confer advantage in food-scarce environments but create risk in western food-rich environments (Barker 1995; Bateson et al. 2004). Low birth weight is also associated with adolescent depression in the presence of childhood adversities, consistent with the hypothesis (Costello et al. 2007).

A study by Murgatroyd and colleagues found interactive effects of prenatal and postnatal maternal depression on NR3C1 1-F promoter methylation, in young children; specifically, infants of mothers with low prenatal depression were vulnerable to the effects of postnatal depression, at the level of DNA methylation - consistent with an interplay between prenatal and postnatal environments (Murgatroyd et al. 2015). Further work would be important to address mechanisms, especially those genes impacted by prenatal stress that might confer resistance to later effects of postnatal stress.

\section{Epigenetic programming by early-life care}

Variations in parental investment in mammals commonly involve the quantity and quality of behavioral interactions between parent and offspring. Numerous studies highlight the importance of maternal influences on subsequent performance both in terms of physiological functions and behavior of the offspring (Babb et al. 2015). Importantly, the nature of mother- 
offspring interaction influences gene expression and the development of behavioural responses in the offspring, which remain stable from early development to adulthood.

One seminal animal study investigated the epigenetic effects of early life environment on stress programming through variations in the quality of early postnatal maternal care, as measured by levels of licking and grooming. Rats who received high levels of maternal care during early life developed sustained elevations in Nr3c1 expression within the hippocampus and reduced HPA axis responses to stress. Meaney and colleagues further found an important role for epigenetic regulation revealing that the enhanced $\mathrm{Nr} 3 \mathrm{c} 1$ expression associated with a persistent DNA hypomethylation at specific $\mathrm{CpG}$ dinucleotides within the hippocampal Nr3c1 exon 17 promoter and increased histone acetylation. The lower CpG methylation facilitated binding of the transcriptional activator nerve growth factor-inducible protein A (NGF1a) to this region (Weaver et al. 2004).

Two recent studies have reported moderation of prenatal stress effects by early human tactile stimulation (assessed by how often mothers reported stroking their babies) over the first weeks of life on behavioural and physiological stress reactivity in infancy (Pickles et al. 2017; Sharp et al. 2014) demonstrating that the effect of maternal prenatal depression on infant reactivity would be modified by tactile. This was followed up by another study showing a reduction of $N R 3 C 1$ gene methylation associated with maternal stroking on these children, supporting the role of epigenetic mechanisms linking early life stress with long-term effects of maternal care (Murgatroyd et al. 2015).

Recent research is demonstrating that the rewarding sensation of touch in affiliative interactions could be underpinned by a specialized system of nerve fibers called C-Tactile afferents (CTs), which respond optimally to slowly moving, gentle touch, typical of a caress (Pawling et al. 2017; McGlone et al. 2014). Interestingly, studies in rodent have shown that that tactile stimuli activate hypothalamic oxytocin neurons (Anon 2015). This together with 
other recent research suggests $\mathrm{C}$-tactile afferent stimulation may mediate oxytocin release during affiliative tactile interactions (Walker et al. 2017).

\section{Transgenerational effects of stress}

Numerous studies show that children of depressed parents are at risk for developing depressive disorders themselves and other internalizing and externalizing disorders (National Research Council (US) and Institute of Medicine (US) Committee on Depression et al. 2009; Lyons-Ruth et al. 1997), whether this be through prenatal or postnatal exposure to maternal stress. However, whether these risks are transmitted beyond 2 generations is less well known with only few published 3-generation studies of major depression (Pettit et al. 2008; Olino et al. 2008). One study of 800 depressed and never-depressed women together with information from their children (at 15yrs) and grandmothers detected an intergenerational transmission of depression, whereby the grandmother's depression affected the mother's depression and her own stressful life context, and maternal and grandmother depression affected youth depression. This was mediated by interpersonal stress processes, which in turn affected parenting and children's social functioning (Hammen et al. 2004). A further recent study on 251 sets of grandchildren, parents and grandparents found those with 2 previous generations affected with major depression were at highest risk for major depression; though the study was too small to test for sex effects (Weissman et al. 2016).

Factors contributing to transgenerational transmission of depression have been difficult to identify: most studies based on clinical samples either represent unusually adverse contexts that are difficult to generalize and, for the most part, have not been large enough, with multiple collected environmental and biological variables, ideally longitudinally. A recent study by Murgatroyd and Nephew investigated the effects of chronic social stress during lactation in F0 mothers (Murgatroyd \& Nephew 2013) across 3 generations and found that maternal care behavior in the F2 dams was more severely impaired than in the F0 and F1 
dams and the expression of maternal anxiety is augmented in F2 dams. This occurred together with reduced basal cortisol (in contrast to an increase in F1 dams), a lack of changes in neuroendocrine gene expression, and reduced serum ICAM-1 (intercellular adhesion molecule-1) levels - a marker for inflammation and blood-brain barrier integrity (Nephew, Carini, Sallah, Alyamani, Bradburn and Murgatroyd, in submission). The data support the hypothesis that the effects of chronic social stress can accumulate across three generations suggesting mechanisms underlying evolutionary adaption.

\section{Blood epigenetic markers for early-life stress}

Access to brain tissues is obviously limited in human subjects. As such, many studies on specific epigenetic pathways in developmental programming of adult physical and mental problems by early-life stressful conditions have been preformed on peripheral tissue samples. Studies using peripheral DNA, from blood or saliva of infants, adolescents or adults, have shown increased levels of $N R 3 C 1$ methylation in response to perinatal stress (Oberlander et al. n.d.; KM et al. 2011; CJ et al. 2012; Murgatroyd et al. 2015) and abuse or neglect during childhood (Perroud et al. 2011; AR et al. 2012). Most studies thus far reported on DNA methylation after enduring stress or traumatic events such as abuse or neglect (McGowan et al. 2009; Perroud et al. 2011; Perroud et al. 2013; AR et al. 2012). Interestingly, many of these studies have looked at different regions and CpG residues of the NR3C1 1-F promoter regions, though several recent clinical studies examining leukocytes have reported elevated methylation of the homologous human NR3C1 1-F promoter (homologous to the rat 1-7 promoter) at a specific $\mathrm{CpG}$ associated with prenatal maternal depression (Oberlander et al. n.d.; Conradt et al. 2013; Hompes et al. 2013) and childhood stress (AR et al. 2012; PA et al. 2013). This suggests perhaps, different CpG residues might be sensitive to specific environmental stressors across this region (Smart et al. 2015).

This raises the question of whether epigenetic changes in blood provide a clinically valuable surrogate to what is happening in the brain. Initial studies in rhesus macaques by Provencal et al., (Provencal et al. 2012) examining DNA methylation in the prefrontal cortex and 
peripheral T cells in parallel in response to differential rearing did reveal differences in common between both tissues. Though results as a whole tended to suggest the $T$ cells were not a direct surrogate markers of the brain tissue, but probably reflected the response of the immune system to early life stress. However, the idea that the epigenomic response to early life stress is not limited to the brain and could be studied in peripheral lymphocytes is a major area of study.

\section{Perspectives}

There is increasing evidence that exposure to stressful events during prenatal and postnatal periods associates with long-lasting mental health problems. While molecular basis for such long-term effects may not be entirely unclear, much evidence supports a role for the involvement of epigenetic regulation. The idea is that epigenetic tuning in response to early life environment may prepare genes for responses to future environment, i.e. enabling the organism to adapt to changing environmental conditions. These adaptive processes can, however, enhance the risk of pathology in the later life especially if thee is mismatch between early and later life nevironments. Thereby, epigenetic research promises to improve the understanding of the genes and pathways by which organisms show widely diverse responses to stressful events in early life. Several specific genes linked to GR regulation such as NR3C1 are proving to be important markers.

With the advent of new high-throughput sequencing technologies, research is now scaling up to epigenome-wide analyses of all epigenetic marks throughout a genome in a specific tissue. Given that epigenetic modifications are sensitive to changes in the environment, it might be anticipated that these efforts will identify epigenomic signatures for mental disorders and molecular dysregulations resulting from early life stress (Albert, 2010). Whilst such a strategy appears highly attractive in the field of cancer research addressing clonally expanded cell populations, rather than heterogeneous tissues, the field of epigenetic 
association studies in neuroendocrine disorders and psychiatry might be more challenging. Epigenetic modifications reported to date in animal models and postmortem brain tissues are seldom "all or none" but gradual, and seem to occur in a highly cell-type-specific manner. In this view, the extraordinary complexity and heterogeneity of neural tissues poses a major hurdle to derive epigenetic biomarkers in psychiatric disease.

- Taken together we have covered the importance of epigenetic processes in programming long-term neuroendrocrine regulation in response to the prenatal and postnatal environment. Adversity during these early periods is able to shape this towards dysregulation of the HPA axis.

- Epigenetic mechanisms appear key to allowing an organism to respond to the environment through changes in gene expression.

- Numerous studies of early life stress in animal models are revealing the importance of the epigenetic regulation of key neuroendocrine genes. Some clinical studies demonstrate translational aspects of this research. The role of epigenetic marks in peripheral tissues as a convenient surrogate marker for the brain needs to be further explored.

- Overall, findings from these investigations form the hypothesis that early adversityinduced changes in the levels of methylation of the GR gene may disturb neuroendocrine systems, resulting in altered cortisol production, and leading, in turn, to the development of various pathological conditions in adult life.

In sum, understanding how early life experiences can give rise to lasting epigenetic memories conferring increased risk for mental disorders is emerging at the epicenter of modern psychiatry. Whether suitable social or pharmacological interventions could reverse deleterious epigenetic programming triggered by adverse conditions during early life, should receive highest priority on future research agendas. Progress in this field will further garner public interest, a general understanding and appreciation of the consequences of childhood abuse and neglect for victims in later life. 


\section{References}

Anon, 2015. Activation of hypothalamic oxytocin neurons following tactile stimuli in rats. Neuroscience Letters, 600, pp.22-27. Available at: http://www.sciencedirect.com/science/article/pii/S030439401500422X?via\%3Dihub [Accessed September 20, 2017].

Appleton, A.A. et al., 2015. Examining the joint contribution of placental NR3C1 and HSD11B2 methylation for infant neurobehavior. Psychoneuroendocrinology, 52, pp.3242. Available at: http://linkinghub.elsevier.com/retrieve/pii/S0306453014004156 [Accessed November 24, 2016].

AR, T. et al., 2012. Childhood adversity and epigenetic modulation of the leukocyte glucocorticoid receptor: preliminary findings in healthy adults. PLoS One, 7, p.e30148. Available at: http://dx.doi.org/10.1371/journal.pone.0030148.

Babb, J.A. et al., 2015. Peripartum depression and anxiety as an integrative cross domain target for psychiatric preventative measures. Behavioural brain research, 276, pp.3244. Available at: http://www.ncbi.nlm.nih.gov/pubmed/24709228 [Accessed June 22, 2016].

Babenko, O., Kovalchuk, I. \& Metz, G.A.S., 2015. Stress-induced perinatal and transgenerational epigenetic programming of brain development and mental health. Neuroscience \& Biobehavioral Reviews, 48, pp.70-91. Available at: http://linkinghub.elsevier.com/retrieve/pii/S0149763414003091 [Accessed November 24, 2016].

Barker, D.J., 1995. Fetal origins of coronary heart disease. BMJ, 311, pp.171-174. Available at: http://dx.doi.org/10.1136/bmj.311.6998.171.

Bateson, P. et al., 2004. Developmental plasticity and human health. Nature, 430, pp.419421. Available at: http://dx.doi.org/10.1038/nature02725. 
Bertram, C. et al., 2001. The Maternal Diet during Pregnancy Programs Altered Expression of the Glucocorticoid Receptor and Type $211 \beta$-Hydroxysteroid Dehydrogenase: Potential Molecular Mechanisms Underlying the Programming of Hypertension in Utero 1. Endocrinology, 142(7), pp.2841-2853. Available at: http://press.endocrine.org/doi/10.1210/endo.142.7.8238 [Accessed November 24, 2016].

CJ, M. et al., 2012. Methylation changes at NR3C1 in newborns associate with maternal prenatal stress exposure and newborn birth weight. Epigenetics, 7, pp.853-857. Available at: http://dx.doi.org/10.4161/epi.21180.

Conradt, E. et al., 2013. The roles of DNA methylation of NR3C1 and $11 \beta-H S D 2$ and exposure to maternal mood disorder in utero on newborn neurobehavior. Epigenetics, 8(12), pp.1321-1329. Available at: http://www.tandfonline.com/doi/abs/10.4161/epi.26634 [Accessed November 24, 2016].

Costello, E. et al., 2007. Prediction from low birth weight to female adolescent depression: a test of competing hypotheses. Arch Gen Psychiatry, 64, pp.338-344. Available at: http://dx.doi.org/10.1001/archpsyc.64.3.338.

Doherty, T.S. \& Roth, T.L., 2016. Insight from animal models of environmentally driven epigenetic changes in the developing and adult brain. Development and Psychopathology, 28(4pt2), pp.1229-1243. Available at: http://www.journals.cambridge.org/abstract_S095457941600081X [Accessed November 8, 2016].

Gomez, S. et al., 2016. Comparative Analysis of Iron Homeostasis in Sub-Saharan African Children with Sickle Cell Disease and Their Unaffected Siblings. Frontiers in Pediatrics, 4. Available at: http://journal.frontiersin.org/Article/10.3389/fped.2016.00008/abstract [Accessed July 2, 2016]. 
Gonzalez, A., 2013. The impact of childhood maltreatment on biological systems: Implications for clinical interventions. Paediatrics \& child health, 18(8), pp.415-8. Available at: http://www.ncbi.nlm.nih.gov/pubmed/24426793 [Accessed November 25, 2016].

Hammen, C., Shih, J.H. \& Brennan, P.A., 2004. Intergenerational transmission of depression: test of an interpersonal stress model in a community sample. Journal of consulting and clinical psychology, 72(3), pp.511-22. Available at: http://www.ncbi.nlm.nih.gov/pubmed/15279534 [Accessed August 18, 2016].

Heim, C. \& Binder, E.B., 2012. Current research trends in early life stress and depression: Review of human studies on sensitive periods, gene-environment interactions, and epigenetics. Experimental Neurology, 233(1), pp.102-111. Available at: http://linkinghub.elsevier.com/retrieve/pii/S0014488611004043 [Accessed November $25,2016]$.

Holsboer, F., 2000. The Corticosteroid Receptor Hypothesis of Depression. Neuropsychopharmacology, 23(5), pp.477-501. Available at: http://www.nature.com/doifinder/10.1016/S0893-133X(00)00159-7 [Accessed November 7, 2016].

Hompes, T. et al., 2013. Investigating the influence of maternal cortisol and emotional state during pregnancy on the DNA methylation status of the glucocorticoid receptor gene (NR3C1) promoter region in cord blood. J Psychiatr Res, 47, pp.880-891. Available at: http://dx.doi.org/10.1016/j.jpsychires.2013.03.009.

Jensen Peña, C., Monk, C. \& Champagne, F.A., 2012. Epigenetic Effects of Prenatal Stress on 11ß-Hydroxysteroid Dehydrogenase-2 in the Placenta and Fetal Brain K. Sun, ed. PLoS ONE, 7(6), p.e39791. Available at: http://dx.plos.org/10.1371/journal.pone.0039791 [Accessed November 24, 2016]. 
Klengel, T. et al., 2012. Allele-specific FKBP5 DNA demethylation mediates gene-childhood trauma interactions. Nature Neuroscience, 16(1), pp.33-41. Available at: http://www.nature.com/doifinder/10.1038/nn.3275 [Accessed November 25, 2016].

$\mathrm{KM}$, R. et al., 2011. Transgenerational impact of intimate partner violence on methylation in the promoter of the glucocorticoid receptor. Transl Psychiatry, 1, p.e21. Available at: http://dx.doi.org/10.1038/tp.2011.21.

Lyons-Ruth, K., Easterbrooks, M.A. \& Cibelli, C.D., 1997. Infant attachment strategies, infant mental lag, and maternal depressive symptoms: predictors of internalizing and externalizing problems at age 7. Developmental psychology, 33(4), pp.681-92. Available at: http://www.ncbi.nlm.nih.gov/pubmed/9232383 [Accessed August 12, 2016].

McGlone, F., Wessberg, J. \& Olausson, H., 2014. Discriminative and affective touch: sensing and feeling. Neuron, 82(4), pp.737-55. Available at: http://linkinghub.elsevier.com/retrieve/pii/S0896627314003870 [Accessed September 20, 2017].

McGowan, P.O. et al., 2009. Epigenetic regulation of the glucocorticoid receptor in human brain associates with childhood abuse. Nature Neuroscience, 12(3), pp.342-348. Available at: http://www.nature.com/doifinder/10.1038/nn.2270 [Accessed November 24, 2016].

McGowan, P.O. et al., 2008. Promoter-Wide Hypermethylation of the Ribosomal RNA Gene Promoter in the Suicide Brain J. Hoheisel, ed. PLoS ONE, 3(5), p.e2085. Available at: http://dx.plos.org/10.1371/journal.pone.0002085 [Accessed November 7, 2016].

Murgatroyd, C. et al., 2009. Dynamic DNA methylation programs persistent adverse effects of early-life stress. Nature Neuroscience, 12(12), pp.1559-U108.

Murgatroyd, C. et al., 2015. Effects of prenatal and postnatal depression, and maternal 
stroking, at the glucocorticoid receptor gene. Translational psychiatry, 5, p.e560.

Available at: http://www.ncbi.nlm.nih.gov/pubmed/25942041 [Accessed June 22, 2016].

Murgatroyd, C. \& Bradburn, S., 2016. Translational Animal Models for the Study of Epigenetics and the Environment. In Epigenetics, the Environment, and Children's Health Across Lifespans. Cham: Springer International Publishing, pp. 207-229. Available at: http://link.springer.com/10.1007/978-3-319-25325-1_8 [Accessed November 24, 2016].

Murgatroyd, C. \& Spengler, D., 2011a. Epigenetic programming of the HPA axis: early life decides. Stress (Amsterdam, Netherlands), 14(6), pp.581-9. Available at: http://www.ncbi.nlm.nih.gov/pubmed/21854166 [Accessed June 22, 2016].

Murgatroyd, C. \& Spengler, D., 2011b. Epigenetics of Early Child Development. Frontiers in Psychiatry, 2, p.16. Available at:

http://journal.frontiersin.org/article/10.3389/fpsyt.2011.00016/abstract [Accessed November 7, 2016].

Murgatroyd, C. \& Spengler, D., 2011c. Epigenetics of early child development. Frontiers in psychiatry, 2, p.16. Available at: http://www.ncbi.nlm.nih.gov/pubmed/21647402 [Accessed June 22, 2016].

Murgatroyd, C. \& Spengler, D., 2014. Polycomb binding precedes early-life stress responsive DNA methylation at the Avp enhancer. PloS one, 9(3), p.e90277. Available at: http://www.ncbi.nlm.nih.gov/pubmed/24599304 [Accessed June 22, 2016].

Murgatroyd, C.A. \& Nephew, B.C., 2013. Effects of early life social stress on maternal behavior and neuroendocrinology. Psychoneuroendocrinology, 38(2), pp.219-228. Available at: http://linkinghub.elsevier.com/retrieve/pii/S0306453012002132 [Accessed August 9, 2016].

National Research Council (US) and Institute of Medicine (US) Committee on Depression, 
P.P. and the H.D. of C., England, M.J. \& Sim, L.J., 2009. Depression in Parents, Parenting, and Children, National Academies Press (US). Available at:

http://www.ncbi.nlm.nih.gov/pubmed/25009931 [Accessed August 12, 2016].

O'Donnell, K.J. et al., 2012. Maternal prenatal anxiety and downregulation of placental 11ßHSD2. Psychoneuroendocrinology, 37(6), pp.818-826. Available at:

http://linkinghub.elsevier.com/retrieve/pii/S0306453011002848 [Accessed November 24, 2016].

Oberlander, T.F. et al., Prenatal exposure to maternal depression, neonatal methylation of human glucocorticoid receptor gene (NR3C1) and infant cortisol stress responses. Epigenetics, 3(2), pp.97-106. Available at:

http://www.ncbi.nlm.nih.gov/pubmed/18536531 [Accessed November 24, 2016].

Olino, T.M. et al., 2008. Influence of parental and grandparental major depressive disorder on behavior problems in early childhood: a three-generation study. Journal of the American Academy of Child and Adolescent Psychiatry, 47(1), pp.53-60. Available at: http://www.ncbi.nlm.nih.gov/pubmed/18174825 [Accessed August 18, 2016].

PA, M. et al., 2013. Genetic and epigenetic associations of MAOA and NR3C1 with depression and childhood adversities. Int J Neuropsychopharmacol, 1, pp.1-16.

Pawling, R. et al., 2017. C-tactile afferent stimulating touch carries a positive affective value F. Tremblay, ed. PLOS ONE, 12(3), p.e0173457. Available at: http://dx.plos.org/10.1371/journal.pone.0173457 [Accessed September 20, 2017].

Perroud, N. et al., 2013. Childhood maltreatment and methylation of the glucocorticoid receptor gene NR3C1 in bipolar disorder. Br J Psychiatry, 204, pp.30-35. Available at: http://dx.doi.org/10.1192/bjp.bp.112.120055.

Perroud, N. et al., 2011. Increased methylation of glucocorticoid receptor gene (NR3C1) in adults with a history of childhood maltreatment: a link with the severity and type of 
trauma. Trans/ Psychiatry, 1, p.e59. Available at: http://dx.doi.org/10.1038/tp.2011.60.

Pettit, J.W. et al., 2008. Intergenerational transmission of internalizing problems: effects of parental and grandparental major depressive disorder on child behavior. Journal of clinical child and adolescent psychology : the official journal for the Society of Clinical Child and Adolescent Psychology, American Psychological Association, Division 53, 37(3), pp.640-50. Available at: http://www.ncbi.nlm.nih.gov/pubmed/18645754 [Accessed August 18, 2016].

Pickles, A. et al., 2017. Prenatal anxiety, maternal stroking in infancy, and symptoms of emotional and behavioral disorders at 3.5 years. European Child \& Adolescent Psychiatry, 26(3), pp.325-334. Available at: http://www.ncbi.nlm.nih.gov/pubmed/27464490 [Accessed May 3, 2017].

Provencal, N. et al., 2012. The Signature of Maternal Rearing in the Methylome in Rhesus Macaque Prefrontal Cortex and T Cells. Journal of Neuroscience, 32(44), pp.1562615642. Available at: http://www.jneurosci.org/cgi/doi/10.1523/JNEUROSCI.147012.2012 [Accessed November 26, 2016].

Seckl, J.R., 2001. Glucocorticoid programming of the fetus; adult phenotypes and molecular mechanisms. Molecular and cellular endocrinology, 185(1-2), pp.61-71. Available at: http://www.ncbi.nlm.nih.gov/pubmed/11738795 [Accessed November 24, 2016].

Sharp, H. et al., 2014. Maternal antenatal anxiety, postnatal stroking and emotional problems in children: outcomes predicted from pre- and postnatal programming hypotheses. Psychol Med, 28, pp.1-15.

Smart, C. et al., 2015. Early life trauma, depression and the glucocorticoid receptor gene--an epigenetic perspective. Psychological medicine, 45(16), pp.3393-410. Available at: http://www.ncbi.nlm.nih.gov/pubmed/26387521 [Accessed June 22, 2016].

Stewart, P.M., Rogerson, F.M. \& Mason, J.I., 1995. Type 211 beta-hydroxysteroid 
dehydrogenase messenger ribonucleic acid and activity in human placenta and fetal membranes: its relationship to birth weight and putative role in fetal adrenal steroidogenesis. The Journal of Clinical Endocrinology \& Metabolism, 80(3), pp.885890. Available at: http://press.endocrine.org/doi/10.1210/jcem.80.3.7883847 [Accessed November 24, 2016].

Togher, K.L. et al., 2014. Epigenetic regulation of the placental HSD11B2 barrier and its role as a critical regulator of fetal development. Epigenetics, 9(6), pp.816-822. Available at: http://www.tandfonline.com/doi/abs/10.4161/epi.28703 [Accessed November 24, 2016].

Walker, S.C. et al., 2017. C-tactile afferents: Cutaneous mediators of oxytocin release during affiliative tactile interactions? Neuropeptides, 64, pp.27-38. Available at: http://linkinghub.elsevier.com/retrieve/pii/S0143417917300112 [Accessed September 20, 2017].

Weaver, I.C.G. et al., 2004. Epigenetic programming by maternal behavior. Nature Neuroscience, 7(8), pp.847-854. Available at: http://www.nature.com/doifinder/10.1038/nn1276 [Accessed November 24, 2016].

Weissman, M.M. et al., 2016. A 30-Year Study of 3 Generations at High Risk and Low Risk for Depression. JAMA Psychiatry, 73(9), p.970. Available at: http://archpsyc.jamanetwork.com/article.aspx?doi=10.1001/jamapsychiatry.2016.1586 [Accessed November 25, 2016].

Welberg, L.A.M., 2005. Chronic maternal stress inhibits the capacity to up-regulate placental 11 -hydroxysteroid dehydrogenase type 2 activity. Journal of Endocrinology, 186(3), pp.R7-R12. Available at: http://joe.endocrinologyjournals.org/cgi/doi/10.1677/joe.1.06374 [Accessed November 24, 2016]. 


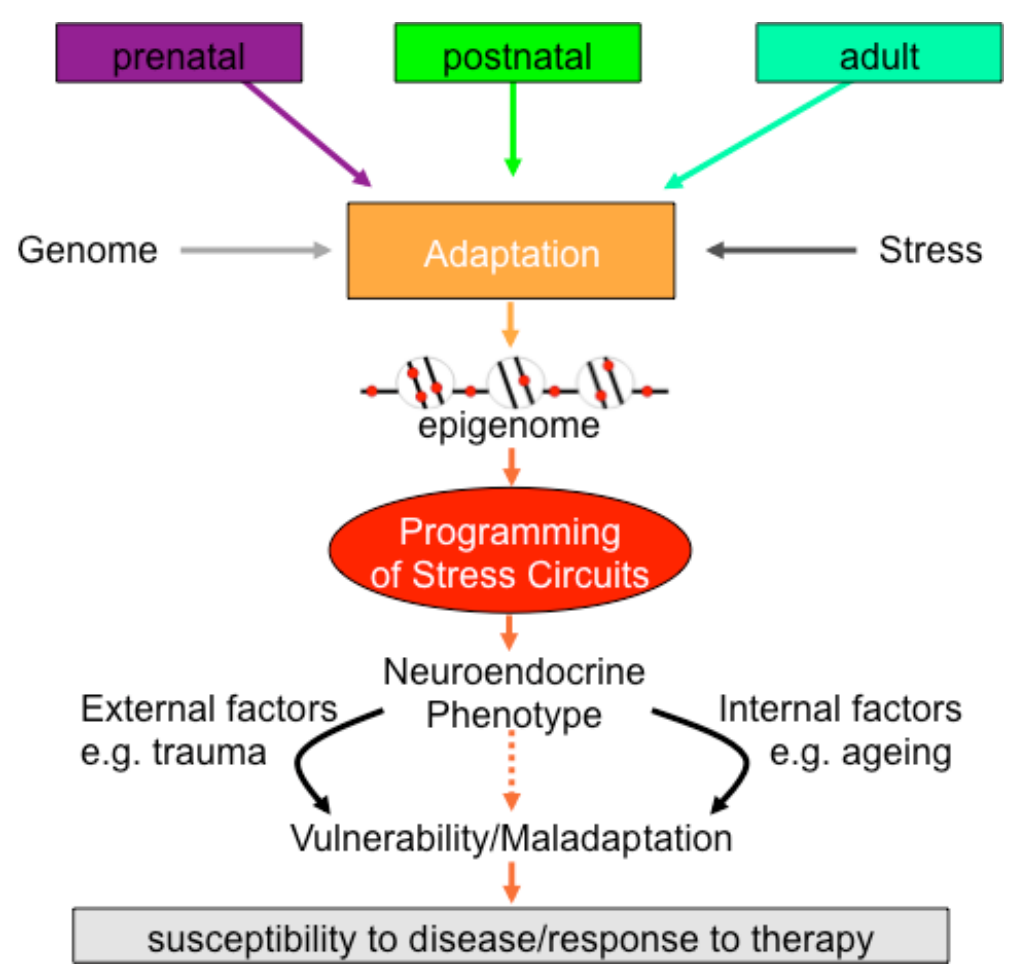

Figure 1. Epigenetic programming by early-life stress. Early life experience can program behaviour, neuroendocrine and stress responsivity throughout later life persistently through alteration of the expression levels of key genes involved in stress regulation through epigenetic marking. The nature of the environment throughout later life, may exacerbate the effects of programming established during early life resulting in increased vulnerability to mood disorders. 